\title{
What can Machine Learning do for Radio Spectrum Management?
}

\author{
Ebtesam Almazrouei \\ Emirates ICT Innovation Centre \\ Khalifa University of Science and Technology \\ Abu Dhabi, UAE \\ ebtesam.almazrouei@ku.ac.ae \\ Nawaf Almoosa \\ Emirates ICT Innovation Centre \\ Khalifa University of Science and Technology \\ Abu Dhabi, UAE \\ nawaf.almoosa@ku.ac.ae
}

\author{
Gabriele Gianini \\ Emirates ICT Innovation Centre \\ Khalifa University of Science and Technology \\ Dip. Informatica, Università degli Studi di Milano \\ Milano, Italy \\ gabriele.gianini@unimi.it \\ Ernesto Damiani \\ Research Centre on Cyber-Physical Systems (C2PS), \\ Khalifa University of Science and Technology \\ Dip. Informatica, Università degli Studi di Milano \\ Milano, Italy \\ ernesto.damiani@unimi.it
}

\begin{abstract}
The opening of the unlicensed radio spectrum creates new opportunities and new challenges for communication technology that can be faced by Machine Learning techniques. In this work, we discuss the potential benefits and the challenges with reference to the recent research developments in this area. Applications go from channel estimation to Signal quality control, and from signal classification to action control. We survey Machine learning and Deep Learning algorithms with possible radio applications, and highlight the corresponding challenges.
\end{abstract}

\section{CCS CONCEPTS}

- Networks $\rightarrow$ Wireless access networks; • Computing methodologies $\rightarrow$ Machine learning.

\section{KEYWORDS}

Radio signals; Wireless Communication; Machine learning

\section{ACM Reference Format:}

Ebtesam Almazrouei, Gabriele Gianini, Nawaf Almoosa, and Ernesto Damiani. 2020. What can Machine Learning do for Radio Spectrum Management?. In 16th ACM Symposium on QoS and Security for Wireless and Mobile Networks (Q2SWinet'20), November 16-20, 2020, Alicante, Spain. ACM, New York, NY, USA, 7 pages. https://doi.org/10.1145/3416013.3426443

Permission to make digital or hard copies of all or part of this work for personal or classroom use is granted without fee provided that copies are not made or distributed for profit or commercial advantage and that copies bear this notice and the full citation on the first page. Copyrights for components of this work owned by others than ACM must be honored. Abstracting with credit is permitted. To copy otherwise, or republish, to post on servers or to redistribute to lists, requires prior specific permission and/or a fee. Request permissions from permissions@acm.org.

Q2SWinet'20, November 16-20, 2020, Alicante, Spain

(C) 2020 Association for Computing Machinery.

ACM ISBN 978-1-4503-8120-8/20/11 . \$15.00

https://doi.org/10.1145/3416013.3426443

\section{INTRODUCTION}

The adoption of intelligent techniques in the management of spectrum sharing can support the coexistence of heterogeneous radioaccess technologies and the significantly improve capacity and spectrum utilization. However it has to face several key challenges: the effectiveness of the algorithms is required to generalize across radio-access scenarios; furthermore, the adopted solutions must be easily applicable across multiple radio standards.

Machine learning (ML) methods, and, more specifically, a set of recently developed techniques, known as Deep Learning (DL) [1], bear the potential of advancing the intelligence of radio devices, providing data-driven flexible solutions, without relying heavily on expert knowledge. Among the problems that the ML can target are protocol detection, and classification, and signal denoising; further applications might include device or user profiling and classification, and source counting.

The present paper is structured as follows: Section II recalls the definitions of ML, DL, and the related work of ML applications to radio signal processing. In section III, the state of art of ML in radio signals is detailed. The related work of applied ML models in Wireless Local Area Network (WLAN) and Fifth Generation (5G) are discussed are discussed in Section IV. Section V detailed the DL models which are applied for radio signals. Conclusions are drawn and in Section VI.

\section{MACHINE LEARNING FOR RADIO SIGNALS}

ML is developed to create an algorithm which can find regularities in a dataset. ML has to execute task $T$ where the goal is to train the network to achieve task $T$ while maintaining a particular performance metric $P$. The system will improve $P$ while training the ML network towards task T. ML models are classified to supervised machine learning or unsupervised machine learning. In supervised machine learning, a labeled dataset is required to generate a general hypothesis about the distribution of class labels to be used as predictor/classifier. The resulting classifier aims to assign a class label to a testing dataset where the value of the data is 
known, but the value of the class label is not [33]. The K-nearest neighbor (KNN) [72], support vector machine(SVM), and Bayesian learning [44] are supervised ML models [26].

For unsupervised ML, the classes of training dataset are not labeled and there are no correct answers to guide the training process. Unsupervised algorithms include clustering where the algorithm will group the trained data into sub-clusters. The group of the dataset within the same sub-clusters are assumed to have the same measure of regularities. Another application is unsupervised ML for dimensionality reduction where a new representation is learned for the trained dataset [31]. Then, the new representation can be used as a threshold to detect or eliminate irrelevant information in the testing dataset. Moreover, it is a useful tool for anomaly detection. Unsupervised ML models are k-means clustering [25], independent component analysis (ICA) [28], and principal component analysis (PCA) [39]. Reinforcement learning (RL) [62], Deep Neural Networks(DNN), and deep learning (DL) [23] are machine learning models that are applied also for radio signals.

There is a growing interest in ML for radio networks domain which diverse across different research areas to build an intelligent radio receiver system. This intelligent radio receiver will be capable of accessing the radio spectrum, learn the features, optimize the performance, and take action if required [30]. Figure 1 indicates the possible research areas and the existing ML models that are explored towards radio intelligence. ML models are implemented for channel estimation, signal quality improvement, signal classification, and action/control for a radio signal.

\section{STATE OF ART OF MACHINE LEARNING IN RADIO SIGNALS}

ML models are applied and examined in radio signals field for signal classification, device classification, spectrum detection, noise estimation, radio optimization, and anomaly detection. Table 1 below detailed the applied ML models for radio signals, the targeted communication problem, the type of radio network, the end communication area either for communication devices, protocols, or users. It also includes the cited papers for ML in this type of radio communication problem.

\section{STATE OF ART OF MACHINE LEARNING MODELS FOR WLAN AND 5G}

ML models have been applied and studied in the radio signals field. The following contains literature review on the ML models applied for Wireless Local Area Network (WLAN) and Fifth Generation $(5 \mathrm{G})$.

\subsection{ML models for WLAN}

ML models have been explored for radio signals. In [9], Passive Radiometric Device Identification System (PARADIS) is developed to identify different source network interface cards (NICs) of an IEEE 802.11 frames. The radiometric identification is based on modulation analysis and utilizing SVM and KNN as a classifier. This technique is trained for over 130 identical IEEE 802.11 wireless NICs and shows $99 \%$ accuracy. However, this approach requires PARADIS sensors to be integrated with wireless access points.
ML algorithms have been investigated for source localization using time-of-arrival(TOA) information of received signals in urban environments [8]. The Random Forest algorithm is used to examine the ML classification and regression schemes for source localization. Also, Ray tracing program is used to simulate the urban environment and generate the required data for the experiment. Twenty-five thousands of data are used for training, and five thousand are used for testing. Results show that regression performs better than ML classification methods. The source localization using ML regression methods shows that $99.2 \%$ of the test locations have a margin of $12.5 \mathrm{~m}$ accuracy from its actual position [8].

In [36], a software architecture called RFDump is developed for monitoring packets on heterogeneous wireless networks and detecting different protocols such as Zigbee and IEEE 802.11. GNU software [57] is used to generate the packets while Universal software radio peripheral(USRP) device [15] is gathering the streams of packets. RFDump depends on the physical features of the protocol (e.g., time, phase, and frequency) to detect the protocol. This approach claims that it will ease the online computational for signal classification using a neural network.

DOF prototype is a detector built by [27] to extract features or signatures, estimate the type of the radio signal, their spectrum, and spatial parameters such as angle of arrival (AOA). DOF is evaluated for an indoor office environment, and the datasets are generated using the fftw [20,21] library and GnuRadio software [57] in $100 \mathrm{MHz}$ bandwidth for ISM band and four multiple inputs and multiple outputs (MIMO) antennas. SVM decision tree is used in DOF system to classify a different type of signals coming from Zigbee, WiFi, and Microwave devices. DOF achieves 85\% accuracy for OdB SNR which outperform RFdump detector [36].

A semi-supervised ML model is applied in [53] to recognize the radio signal modulation types. The modulation class for a radio signal is generated using GNU to produce RadioML16.04 data [52]. Convolutional Neural Network $(\mathrm{CNN})$ is adapted with non-labeled classes to allow learning new features from the sparse representations of raw sampled radio signal time series examples. The results primes a new way to differentiate or recall new and unknown radio signals without the need for expert guidance. However, there is much work left to have the more robust semi-supervised method for radio modulation. More data should be generated, features should be generalized to unknown modulation classes, and well-defined metrics for a semi-supervised model which may consist of full class confusion matrices and the classification accuracy measures.

\subsection{Models for 5G}

Machine learning algorithms and tools are investigated also for possible applications to $5 \mathrm{G}$ networks. The supervised, un-supervised, and reinforcement learning were explored for $5 \mathrm{G}$ applications including cognitive radios, large-scale MIMOs, device-to- device (D2D) networks, energy harvesting, heterogeneous networks constituted by femto-cells and small-cells, and for smart grid. In [30], the author surveyed ML paradigms for next generation wireless networks. The survey showed that they are supervised learning, un-supervised learning, and reinforcement learning algorithms that are investigated for $5 \mathrm{G}$ applications. 


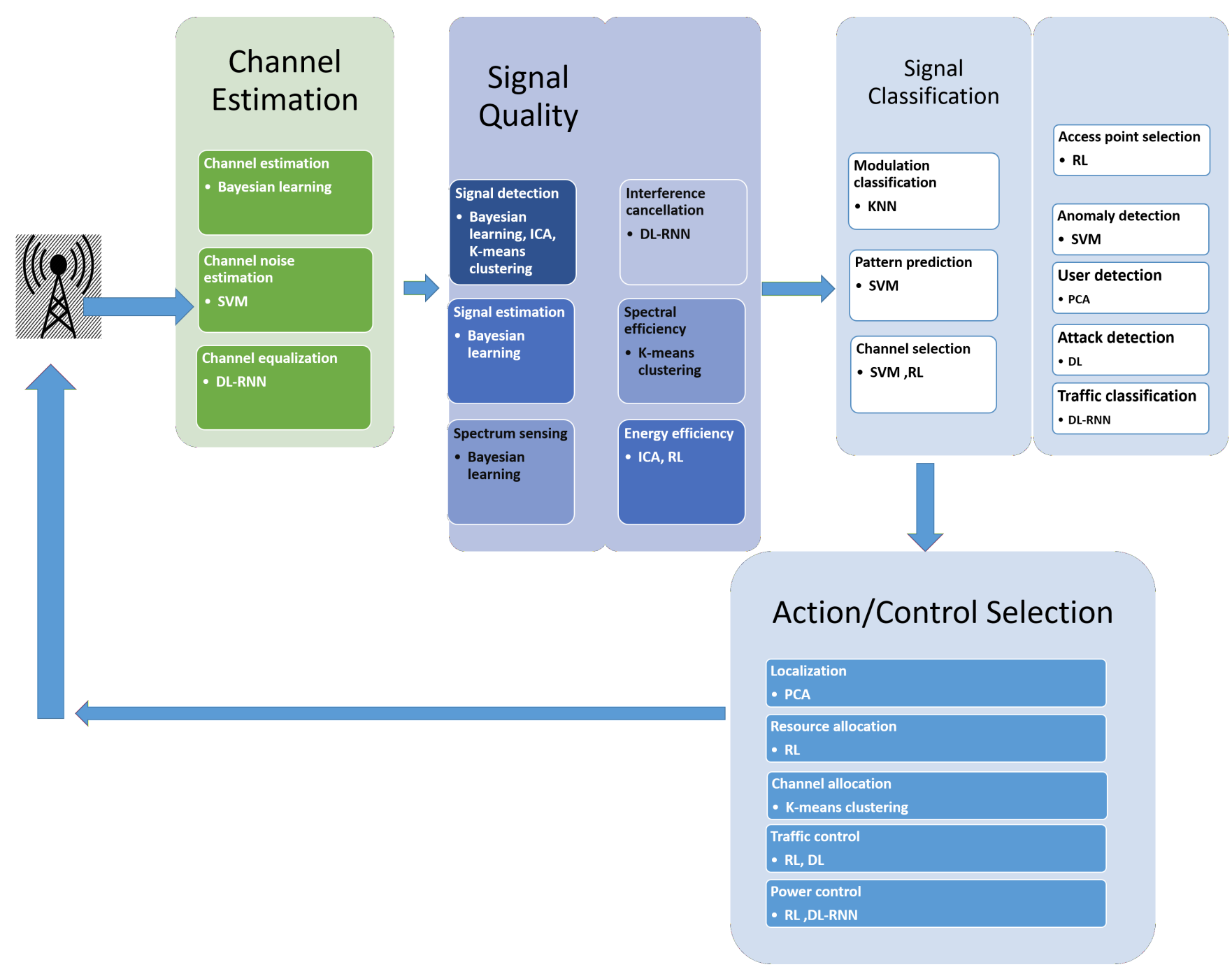

Figure 1: ML models in various radio receiver areas.

Supervised learning consist of regression models, KNN algorithm, Bayesian learning, and SVM which require known labels and models. These known models and parameters are necessary to estimate radio parameters and massive MIMO channel, predict user's pattern, and detect white space spectrum for adaptive filtering detection in cognitive radio from physical and network layer. Moreover, the supervised learning methods are applied for application layer to estimate user behaviors and the current user's location to improve quality of service (QoS) in the network.

The unsupervised learning relies on input data without labelling or identify the class of the input data. Unsupervised learning algorithms which were explored for $5 \mathrm{G}$ are PCA, ICA, and K-means clustering. They were deployed for cell clustering, heterogeneous base station clustering in heterogeneous networks (HetNets), and for the dimensionality reduction for the signal in physical layer of massive MIMO systems. In addition, unsupervised learning can be utilized for fault detection, anomaly detection, intrusion detection problems -based on traffic monitoring in wireless networks, and user's behaviors classification in cognitive radio networks.

The third part of ML algorithms for wireless communication is reinforcement learning [3] which relies on a dynamic iterative learning and decision making process. It is mainly studied in wireless communication using Markov decision processes (MDPs) and partially observable Markov decision process (POMDP) which can be seen as a generalization of MDP. It was deployed for network selection of heterogeneous networks (HetNets), for energy harvesting sensors to find optimal outage power, and for distributed resource allocation under unknown system transition model in femto and small-cell networks. Also, reinforcement learning can improve the mobile users' decision making without any information related to the network conditions and the channel status. It also been utilized for resource allocation in the downlink of femtocells. Table 2 illustrates machine learning models that were investigated for $5 \mathrm{G}$ networks; it shows that applying ML is important 
Table 1: ML models applied for radio signals

\begin{tabular}{|c|c|c|c|c|c|}
\hline ML models & Communication problem & Network & ML Area & $\begin{array}{l}\text { Communication Appli- } \\
\text { cation }\end{array}$ & Author \\
\hline H-SVM & Channel noise estimation & MIMO & Classification & Mobile nodes/users & {$[17]$} \\
\hline H-SVM & Location estimation & $\begin{array}{l}\text { mobile ad hoc net- } \\
\text { works nodes }\end{array}$ & Classification & Mobile nodes/users & {$[17]$} \\
\hline SVM, KNN & $\begin{array}{l}\text { Usage pattern pattern recogni- } \\
\text { tion }\end{array}$ & HetNets & Classification & users & {$[13]$} \\
\hline SVM & Channel selection & Cognitive radio & Classification & Users & {$[63]$} \\
\hline SVM & Spectrum detection & MIMO & Classification & protocols & [27] \\
\hline $\mathrm{KNN}$ & $\begin{array}{l}\text { Radio resource reconfigura- } \\
\text { tion/ Traffic detection }\end{array}$ & Cognitive radio & Optimization & Cells & {$[18]$} \\
\hline $\mathrm{KNN}$ & Anomaly detection & HetNets & Management & Cells & {$[46]$} \\
\hline $\mathrm{KNN}$ & Modulation classification & Cognitive radio & Classification & Users & [6] \\
\hline SVM & Anomaly detection & Sensor networks & Classification & Protocols & {$[37]$} \\
\hline Bayesian learning & Spectrum sensing & Cognitive radio & Optimization & Spectrum & {$[68]$} \\
\hline Bayesian learning & Users detection & Cellular network & Optimization & Users & {$[4]$} \\
\hline Bayesian learning & $\begin{array}{l}\text { signal detection ,noise estima- } \\
\text { tion }\end{array}$ & Cognitive radio & Detection & Users & {$[7]$} \\
\hline Bayesian learning & Channel parameter estimation & MIMO & & & \\
\hline Q-learning & Channel selection & Cognitive radio & Optimization & Spectrum & [58] \\
\hline$\widetilde{C N N}$ & Identification & IEEE 802.15 .4 devices & Identification & Devices & [41] \\
\hline RNN & Image reconstruction & $\begin{array}{ll}\text { Synthetic } & \text { aperture } \\
\text { radar (SAR) } & \end{array}$ & Reconstruction & Spectrum & [69] \\
\hline $\mathrm{CNN}$ & $\begin{array}{l}\text { Spectrum sensing, Modulation } \\
\text { classification }\end{array}$ & Cognitive radio & Classification & Spectrum & {$[54]$} \\
\hline DNN & Modulation classification & Cognitive radio & Classification & Users & {$[32]$} \\
\hline DNN & Modulation classification & Cognitive radio & Classification & Users & [55] \\
\hline DNN & Indoor localization & Wi-Fi signals & Classification & Antennas & [64] \\
\hline
\end{tabular}

research challenge for wireless communication and solving it will optimize the future applications in $5 \mathrm{G}$ networks.

\section{DEEP LEARNING FOR RADIO SIGNALS}

DL is introduced by [23] as an answer to the massive need of intelligent technologies to automate routine operations, make diagnoses in medicine, and understand speech. Deep learning concept allows computers to learn the hierarchy of concepts and understand the dataset without the need for human expertise. DL has an expressive capacity and convenient optimization capability that leads to increased interest to apply it for computer vision and natural language processing. In the following section, the potential benefits of applying DL to radio signal communication are discussed. The challenges of complex communication scenario, unknown channel models, and high speed of processing requirements with sharing complex processing unit develop a need to apply DL for radio signals for secure and efficient radio access.

\subsection{Why Deep Learning in Physical Layer for Radio Signals}

DL is a promising technique to apply in formal mathematical models to a computer and natural language processing to characterize or differentiate real languages or world images. Nowadays, DL models outperform human levels of accuracy of detection algorithms for objects in images or handwritten digits [47]. There are known detection algorithms used in the communication field that capture information from transmitted signals to detect/distinguish between the variety of systems and channel models such as detection the type of constellation in AWGN. However, we agree that implementing DL algorithms in such straight-forward scenarios will not yield significant improvements to the physical layer of communication systems. Rather, we expect DL to improve the performance in complex communication scenarios that are still under research in communication filed to find robust and generalized mathematical models [47] (e.g., detection signals in a harsh environment with low SNR and severe multipath effect). Potential benefits of applying DL models to existing techniques for the physical layer can be summarized as follows:

- Most existing signal processing algorithms for the physical layer in wireless communication systems have well defined statistical information which is of Gaussian, linear, and stationary nature. However, having reliable wireless communication systems in practice suffers from nonlinearities and imperfections due to time-varying and frequency selective [60]. Existing signal processing models can approximately capture the tractable practical problems with certain limitations and of research from wireless communication community is still ongoing to improve them. Thus, having DL as a processing block will allow optimizing the wireless network at least for a specific application or imperfection without the need of formal mathematical models.

- An end-to-end communication system is divided into multiple signal processing blocks, where each one performing a specific task such as coding, modulation, equalization, etc. The overall performance needs to be optimized; however, each block's performance is known to be sub-optimal. For example, the separation between the source and the channel coding in a practical channel (e.g., Rayleigh channel [71]) 
Table 2: Summary of applied machine learning algorithms in 5G.

\begin{tabular}{|c|c|c|c|c|}
\hline Paper & Type of ML & ML model & Target & Application \\
\hline [17] & Supervised & SVM & location estimation & MIMO systems \\
\hline [13] & Supervised & $\begin{array}{l}\text { Regression models and } \\
\text { KNN }\end{array}$ & pattern prediction & Energy learning \\
\hline [65] & Supervised & Bayesian learning & Channel estimation & Massive MIMO \\
\hline [12] & Supervised & Bayesian learning & User detection & Cognitive Radio \\
\hline$[70]$ & Supervised & Bayesian learning & Spectrum detection & Cognitive Radio \\
\hline [7] & Supervised & Bayesian learning & Channel estimation & Cognitive Radio \\
\hline$\overline{\text { [67] }}$ & Un-supervised & K-means clustering & Network optimization & $\begin{array}{l}\text { Heterogeneous net- } \\
\text { works }\end{array}$ \\
\hline [56] & Un-supervised & PCA and ICA & Data recovery & $\begin{array}{l}\text { Smart grid cognitive } \\
\text { networks }\end{array}$ \\
\hline [45] & Un-supervised & ICA & User detection & $\begin{array}{l}\text { Spectrum learning in } \\
\text { cognitive radio }\end{array}$ \\
\hline [5] & $\begin{array}{l}\text { Reinforcement learn- } \\
\text { ing }\end{array}$ & $\begin{array}{l}\text { Markov decision pro- } \\
\text { cesses }\end{array}$ & energy harvesting & $\begin{array}{l}\text { Energy harvesting sen- } \\
\text { sors }\end{array}$ \\
\hline [40] & $\begin{array}{l}\text { Reinforcement learn- } \\
\text { ing }\end{array}$ & Multi-armed bandit & network optimization & $\begin{array}{l}\text { Device-to-device } \\
\text { (D2D) networks }\end{array}$ \\
\hline [2] & $\begin{array}{l}\text { Reinforcement learn- } \\
\text { ing }\end{array}$ & Q-learning & Spectrum allocation a & Femto and small cells \\
\hline [46] & $\begin{array}{l}\text { Reinforcement learn- } \\
\text { ing }\end{array}$ & Q-learning & Resource allocation & $\begin{array}{l}\text { Dense small cells } \\
\text { in heterogeneous } \\
\text { network }\end{array}$ \\
\hline
\end{tabular}

and short block length [22]. In [66], factor graphs are proposed to optimize each block, but the proposed approach requires complex systems and increases the computational cost. Using DL to learn end-to-end system model may optimize the performance without the knowledge of optimal an end-to-end mathematical model.

- Neural networks(NNs) are able to approximate any measurable function to any desired degree of accuracy [19]. Recent studies show that Recurrent NNs (RNN) have a remarkable capacity for learning algorithms with faster execution and at lower energy than the manually programmed counterparts as it could perform with low precision data types and highly parallel concurrent architectures [47].

- Higher level programming languages play an essential role to utilize the massively parallel processing architectures and distributed architectures efficiently. Nowadays, the spreading availability and the cheap cost of the Graphical Processing Units (GPU) and the Field Programmable Gate Arrays (FPGAs) enable training of DL models required for real-time signal processing applications. Therefore, running parallel ML algorithms in GPUs and specialized chips for ML inference such as Eyeriss [11] demonstrates the capability of utilizing NNs for high computational throughput with very efficient energy.

\subsection{State of Art of Deep Learning in Radio Signals}

There is increasing interest in the last years to apply DL to various radio communication disciplines.

5.2.1 DL as an Optimizer Block in Communication Systems . DL is studied for belief propagation (BP). BP is a decoding method which proceeds in iterations of message passing. In [43], DL is implemented in BP for linear error correcting codes for channel decodeing where the neural network decoder improves the Signal to Noise Ratio (SNR) up to $1.5 \mathrm{~dB}$ on standard BP for cycle reduced parity check matrices. Also, a RNN architecture is proposed to enhance the performance of parity check matrices. The network performance improves up to $1.0 \mathrm{~dB}$ with with lower densities and fewer short cycles [42]. However, this approach suffers from high complex real time implementation due to the cost of the huge multiplication. In [38], they propose a new decoding algorithm based on DL decoder for BP which provides feasible path for hardware implementation with less than half the number of multiplication 
and less complexity compared to [42]. Moreover, DNN is proposed for one-shot decoding of random and structured codes. Results showed that neural network performance is similar to the network performance for 16 bit length codes, and the neural network is able to generalize decoding algorithms in the structured codes [24]. In [10], NN sub-blocks for polar codes are applied for non-iterative decoding algorithm which improves BER for DB decoding stages. Also, DL based $\mathrm{NN}$ is applied for decoding a stabilizer quantum error correcting code [34]. The NN decoding algorithm developed in [34] outperforms the traditional decoders (e.g. Minimal-Weight Perfect Matching (MWPM) decoder) and shows that it can be employed to any stabilizer code.

5.2.2 DL as End-to-End Communication Systems. A DL based autoencoder has been proposed over an Additive White Gaussian Noise (AWGN) channel to optimize end-to-end communication system performance for small block code [47]. Also, an end-to-end unsupervised radio transformer model is presented as an end-toend communication system [51]. Convolutional Neural Networks $(\mathrm{CNN})$ is applied to the complex-valued temporal radio signal for modulation recognition. Results show that the CNN model delivers the same results as traditional modulation classification [48]. $\mathrm{CNN}$ is applied for spectrum identification based on modulation recognition using IQ samples. The accuracy was $79 \%$ for high SNR $18 \mathrm{~dB}$ [35]. Moreover, convolutional autoencoder has been implemented for compression of raw radio communication [49]. Supervised learning for MIMO detection is applied in [29]. Also, autoencoder is applied to perform as MIMO channel autoencoder receiver [50] and is proposed as new physical layer design for communication system by NN with AWGN channel [14]. DL based detection models have been investigated for learning of encryption/decryption schemes for an unknown channel model [1]. Finally, DL detection algorithms have been studied for molecular communication where the mathematical channel model is unknown, and the knowledge of the channel is not used [16]. DNNs are trained for wireless resource management to solve complex optimization tasks for real-time wireless resource allocation [61]. Long short term memory (LSTM) is used as deep learning model for signal classification based on automatic modulation recognition using Electrosense sensors[59].

\section{CONCLUSION}

Radio spectrum is increasingly becoming a complex environment driven by devices with nondeterministic spatiotemporal accesses as well as variable transmission powers and frequencies of operation. The need for higher data rates under spectrum scarcity will drive further access-pattern complexity,since devices will be required to dynamically share spectrum resources to increase utilization while ensuring minimal disruption to other users. This paper provides an extensive related work and the state of art of ML and DL models that have been explored for radio signals. It also highlights the various applications that has been addressed in communication by using ML and DL approaches.

\section{REFERENCES}

[1] Martín Abadi and David G Andersen. 2016. Learning to protect communications with adversarial neural cryptography. arXiv preprint arXiv:1610.06918 (2016).
[2] Ghassan Alnwaimi, Seiamak Vahid, and Klaus Moessner. 2015. Dynamic heterogeneous learning games for opportunistic access in lte-based macro/femtocell deployments. IEEE Transactions on Wireless Communications 14, 4 (2015), 22942308.

[3] Ethem Alpaydin. 2014. Introduction to machine learning. MIT press.

[4] Rafhael Amorim, Jeroen Wigard, Huan Nguyen, Istvan Z Kovacs, and Preben Mogensen. 2017. Machine-Learning Identification of Airborne UAV-UEs Based on LTE Radio Measurements. In Globecom Workshops (GC Wkshps), 2017 IEEE. IEEE, 1-6.

[5] Anup Aprem, Chandra R Murthy, and Neelesh B Mehta. 2013. Transmit power control policies for energy harvesting sensors with retransmissions. IEEE fournal of Selected Topics in Signal Processing 7, 5 (2013), 895-906.

[6] Muhammad Waqar Aslam, Zhechen Zhu, and Asoke Kumar Nandi. 2012. Automatic modulation classification using combination of genetic programming and KNN. IEEE Transactions on wireless communications 11, 8 (2012), 2742-2750.

[7] Ayman Assra, Jiaxin Yang, and Benoit Champagne. 2016. An EM Approach for cooperative spectrum sensing in multiantenna CR networks. IEEE Transactions on Vehicular Technology 65, 3 (2016), 1229-1243.

[8] Darcy A Bibb, Zhengqing Yun, and Magdy F Iskander. 2016. Machine learning for source localization in urban environments. In Military Communications Conference, MILCOM 2016-2016 IEEE. IEEE, 401-405.

[9] Vladimir Brik, Suman Banerjee, Marco Gruteser, and Sangho Oh. 2008. Wireless device identification with radiometric signatures. In Proceedings of the 14th ACM international conference on Mobile computing and networking. ACM, 116-127.

[10] Sebastian Cammerer, Tobias Gruber, Jakob Hoydis, and Stephan ten Brink. 2017 Scaling deep learning-based decoding of polar codes via partitioning. In GLOBECOM 2017-2017 IEEE Global Communications Conference. IEEE, 1-6.

[11] Yu-Hsin Chen, Tushar Krishna, Joel S Emer, and Vivienne Sze. 2017. Eyeriss: An energy-efficient reconfigurable accelerator for deep convolutional neural networks. IEEE Journal of Solid-State Circuits 52, 1 (2017), 127-138.

[12] Kae Won Choi and Ekram Hossain. 2013. Estimation of primary user parameters in cognitive radio systems via hidden Markov model. IEEE transactions on signal processing 61, 3 (2013), 782-795.

[13] Brad K Donohoo, Chris Ohlsen, Sudeep Pasricha, Yi Xiang, and Charles Anderson. 2014. Context-aware energy enhancements for smart mobile devices. IEEE Transactions on Mobile Computing 13, 8 (2014), 1720-1732.

[14] Sebastian Dörner, Sebastian Cammerer, Jakob Hoydis, and Stephan ten Brink. 2018. Deep learning based communication over the air. IEEE Journal of Selected Topics in Signal Processing 12, 1 (2018), 132-143.

[15] M Ettus. 2012. Universal software radio peripheral, Ettus Research, Mountain View, CA.

[16] Nariman Farsad and Andrea Goldsmith. 2017. Detection algorithms for communication systems using deep learning. arXiv preprint arXiv:1705.08044 (2017).

[17] Vin-sen Feng and Shih Yu Chang. 2012. Determination of wireless networks parameters through parallel hierarchical support vector machines. IEEE Transactions on Parallel and Distributed Systems 23, 3 (2012), 505-512.

[18] Zhiyong Feng, Xi Li, Qixun Zhang, and Wei Li. 2017. Proactive Radio Resource Optimization With Margin Prediction: A Data Mining Approach. IEEE Transactions on Vehicular Technology 66, 10 (2017), 9050-9060.

[19] R Fierro and FL Lewis. 1999. Multilayer feedforward networks are universa approximators. IEEE Trans. Syst., Man, Cybern 29, 6 (1999), 649-654.

[20] Matteo Frigo and Steven G Johnson. 1999. FFTW user's manual. Massachusetts Institute of Technology (1999).

[21] Matteo Frigo and Steven G Johnson. 2004. The FFTW web page. URL: http://www. fftw. org (2004)

[22] Andrea Goldsmith. 1995. Joint source/channel coding for wireless channels. In Vehicular Technology Conference, 1995 IEEE 45th, Vol. 2. IEEE, 614-618.

[23] Ian Goodfellow, Yoshua Bengio, Aaron Courville, and Yoshua Bengio. 2016. Deep learning. Vol. 1. MIT press Cambridge.

[24] Tobias Gruber, Sebastian Cammerer, Jakob Hoydis, and Stephan ten Brink. 2017. On deep learning-based channel decoding. In Information Sciences and System (CISS), 2017 51st Annual Conference on. IEEE, 1-6.

[25] John A Hartigan and Manchek A Wong. 1979. Algorithm AS 136: A k-means clustering algorithm. Journal of the Royal Statistical Society. Series C (Applied Statistics) 28, 1 (1979), 100-108.

[26] Marti A. Hearst, Susan T Dumais, Edgar Osuna, John Platt, and Bernhard Scholkopf. 1998. Support vector machines. IEEE Intelligent Systems and their applications 13, 4 (1998), 18-28.

[27] Steven Siying Hong and Sachin Rajsekhar Katti. 2011. DOF: a local wireless information plane. In ACM SIGCOMM Computer Communication Review, Vol. 41 ACM, 230-241.

[28] Aapo Hyvärinen and Erkki Oja. 2000. Independent component analysis: algorithms and applications. Neural networks 13, 4-5 (2000), 411-430.

[29] Yo-Seb Jeon, Song-Nam Hong, and Namyoon Lee. 2016. Blind detection for MIMO systems with low-resolution ADCs using supervised learning. arXiv preprint (2016).

[30] Chunxiao Jiang, Haijun Zhang, Yong Ren, Zhu Han, Kwang-Cheng Chen, and Lajos Hanzo. 2017. Machine learning paradigms for next-generation wireless 
networks. IEEE Wireless Communications 24, 2 (2017), 98-105.

[31] Hanna Kamyshanska and Roland Memisevic. 2013. On autoencoder scoring. In International Conference on Machine Learning. 720-728.

[32] Byeoungdo Kim, Jaekyum Kim, Hyunmin Chae, Dongweon Yoon, and Jun Won Choi. 2016. Deep neural network-based automatic modulation classification technique. In Information and Communication Technology Convergence (ICTC), 2016 International Conference on. IEEE, 579-582.

[33] Sotiris B Kotsiantis, I Zaharakis, and P Pintelas. 2007. Supervised machine learning: A review of classification techniques. Emerging artificial intelligence applications in computer engineering 160 (2007), 3-24.

[34] Stefan Krastanov and Liang Jiang. 2017. Deep neural network probabilistic decoder for stabilizer codes. Scientific reports 7, 1 (2017), 11003.

[35] Merima Kulin, Tarik Kazaz, Ingrid Moerman, and Eli De Poorter. 2018. End-toend learning from spectrum data: A deep learning approach for wireless signal identification in spectrum monitoring applications. IEEE Access 6 (2018), 1848418501.

[36] Kaushik Lakshminarayanan, Samir Sapra, Srinivasan Seshan, and Peter Steenkiste. 2009. RFDump: an architecture for monitoring the wireless ether. In Proceedings of the 5th international conference on Emerging networking experiments and technologies. ACM, 253-264.

[37] Zhida Li, Qingye Ding, Soroush Haeri, and Ljiljana Trajković. 2018. Application of machine learning techniques to detecting anomalies in communication networks: Classification algorithms. Cyber Threat Intelligence (2018), 71-92.

[38] Loren Lugosch and Warren J Gross. 2017. Neural offset min-sum decoding. In Information Theory (ISIT), 2017 IEEE International Symposium on. IEEE, 13611365.

[39] Andrzej Mackiewicz and Waldemar Ratajczak. 1993. Principal components analysis (PCA). Computers and Geosciences 19 (1993), 303-342.

[40] Setareh Maghsudi and Sławomir Stańczak. 2015. Channel selection for networkassisted D2D communication via no-regret bandit learning with calibrated forecasting. IEEE Transactions on Wireless Communications 14, 3 (2015), 1309-1322.

[41] Kevin Merchant, Shauna Revay, George Stantchev, and Bryan Nousain. 2018 Deep learning for RF device fingerprinting in cognitive communication networks. IEEE Journal of Selected Topics in Signal Processing 12, 1 (2018), 160-167.

[42] Eliya Nachmani, Elad Marciano, David Burshtein, and Yair Be'ery. 2017. RNN decoding of linear block codes. arXiv preprint arXiv:1702.07560 (2017)

[43] Eliya Nachmani, Elad Marciano, Loren Lugosch, Warren J Gross, David Burshtein, and Yair Be'ery. 2018. Deep learning methods for improved decoding of linear codes. IEEE fournal of Selected Topics in Signal Processing 12, 1 (2018), 119-131.

[44] Radford M Neal. 2012. Bayesian learning for neural networks. Vol. 118. Springer Science \& Business Media.

[45] Huy Nguyen, Guanbo Zheng, Rong Zheng, and Zhu Han. 2013. Binary inference for primary user separation in cognitive radio networks. IEEE Transactions on Wireless Communications 12, 4 (2013), 1532-1542.

[46] Oluwakayode Onireti, Ahmed Zoha, Jessica Moysen, Ali Imran, Lorenza Giupponi, Muhammad Ali Imran, and Adnan Abu-Dayya. 2016. A cell outage management framework for dense heterogeneous networks. IEEE Transactions on Vehicular Technology 65, 4 (2016), 2097-2113.

[47] Timothy O'Shea and Jakob Hoydis. 2017. An introduction to deep learning for the physical layer. IEEE Transactions on Cognitive Communications and Networking 3, 4 (2017), 563-575.

[48] Timothy J O'Shea, Johnathan Corgan, and T Charles Clancy. 2016. Convolutional radio modulation recognition networks. In International conference on engineering applications of neural networks. Springer, 213-226.

[49] Timothy J O'Shea, Johnathan Corgan, and T Charles Clancy. 2016. Unsupervised representation learning of structured radio communication signals. In Sensing, Processing and Learning for Intelligent Machines (SPLINE), 2016 First International Workshop on. IEEE, 1-5.

[50] Timothy J O'Shea, Tugba Erpek, and T Charles Clancy. 2017. Deep learning based MIMO communications. arXiv preprint arXiv:1707.07980 (2017).

[51] Timothy J O'Shea, Kiran Karra, and T Charles Clancy. 2016. Learning to communicate: Channel auto-encoders, domain specific regularizers, and attention. In Signal Processing and Information Technology (ISSPIT), 2016 IEEE International Symposium on. IEEE, 223-228.

[52] Timothy J O'Shea and Nathan West. 2016. Radio machine learning dataset generation with gnu radio. In Proceedings of the GNU Radio Conference, Vol. 1.

[53] Timothy J O'Shea, Nathan West, Matthew Vondal, and T Charles Clancy. 2017. Semi-supervised radio signal identification. In Advanced Communication Technology (ICACT), 2017 19th International Conference on. IEEE, 33-38.

[54] Timothy James OâĂŹShea, Tamoghna Roy, and T Charles Clancy. 2018. Overthe-air deep learning based radio signal classification. IEEE fournal of Selected Topics in Signal Processing 12, 1 (2018), 168-179.

[55] Shengliang Peng, Hanyu Jiang, Huaxia Wang, Hathal Alwageed, Yu Zhou, Marjan Mazrouei Sebdani, and Yu-Dong Yao. 2018. Modulation Classification Based on Signal Constellation Diagrams and Deep Learning. IEEE transactions on neural networks and learning systems 99 (2018), 1-10.
[56] Robert C Qiu, Zhen Hu, Zhe Chen, Nan Guo, Raghuram Ranganathan, Shujie Hou, and Gang Zheng. 2011. Cognitive radio network for the smart grid: Experimental system architecture, control algorithms, security, and microgrid testbed. IEEE Trans. Smart Grid 2, 4 (2011), 724-740.

[57] GNU Radio. 2007. The gnu software radio. Available from World Wide Web: https://gnuradio. org (2007).

[58] Vishnu Raj, Irene Dias, Thulasi Tholeti, and Sheetal Kalyani. 2018. Spectrum access in cognitive radio using a two-stage reinforcement learning approach IEEE Journal of Selected Topics in Signal Processing 12, 1 (2018), 20-34.

[59] Sreeraj Rajendran, Wannes Meert, Domenico Giustiniano, Vincent Lenders, and Sofie Pollin. 2018. Deep Learning Models for Wireless Signal Classification with Distributed Low-Cost Spectrum Sensors. IEEE Transactions on Cognitive Communications and Networking (2018).

[60] Tim Schenk. 2008. RF imperfections in high-rate wireless systems: impact and digital compensation. Springer Science \& Business Media.

[61] Haoran Sun, Xiangyi Chen, Qingjiang Shi, Mingyi Hong, Xiao Fu, and Nikos D Sidiropoulos. 2017. Learning to optimize: Training deep neural networks for wireless resource management. In Signal Processing Advances in Wireless Communications (SPAWC), 2017 IEEE 18th International Workshop on. IEEE, 1-6.

[62] Richard S Sutton and Andrew G Barto. 1998. Introduction to reinforcement learning. Vol. 135. MIT press Cambridge.

[63] Karaputugala G Madushan Thilina, Ekram Hossain, and Dong In Kim. 2016. DCCC-MAC: A dynamic common-control-channel-based MAC protocol for cellular cognitive radio networks. IEEE Transactions on Vehicular Technology 65, 5 (2016), 3597-3613.

[64] Xuyu Wang, Lingjun Gao, Shiwen Mao, and Santosh Pandey. 2015. DeepFi: Deep learning for indoor fingerprinting using channel state information. In Wireless Communications and Networking Conference (WCNC), 2015 IEEE. IEEE, 16661671.

[65] Chao-Kai Wen, Shi Jin, Kai-Kit Wong, Jung-Chieh Chen, and Pangan Ting. 2015 Channel estimation for massive MIMO using Gaussian-mixture Bayesian learning. IEEE Transactions on Wireless Communications 14, 3 (2015), 1356-1368.

[66] Henk Wymeersch. 2007. Iterative receiver design. Cambridge Univ. Press.

[67] Ming Xia, Yasunori Owada, Masugi Inoue, and Hiroaki Harai. 2012. Optical and wireless hybrid access networks: Design and optimization. fournal of Optical Communications and Networking 4, 10 (2012), 749-759.

[68] Yizhen Xu, Peng Cheng, Zhuo Chen, Yonghui Li, and Branka Vucetic. 2018. Mobile Collaborative Spectrum Sensing for Heterogeneous Networks: A Bayesian Machine Learning Approach. IEEE Transactions on Signal Processing (2018).

[69] Bariscan Yonel, Eric Mason, and Birsen Yazıc1. 2018. Deep learning for passive synthetic aperture radar. IEEE Journal of Selected Topics in Signal Processing 12, 1 (2018), 90-103.

[70] Chung-Kai Yu, Kwang-Cheng Chen, and Shin-Ming Cheng. 2010. Cognitive radio network tomography. IEEE Transactions on Vehicular Technology 59, 4 (2010), 1980-1997.

[71] Ephraim Zehavi. 1992. 8-PSK trellis codes for a Rayleigh channel. IEEE Transactions on Communications 40, 5 (1992), 873-884.

[72] Min-Ling Zhang and Zhi-Hua Zhou. 2007. ML-KNN: A lazy learning approach to multi-label learning. Pattern recognition 40, 7 (2007), 2038-2048. 